\title{
Determination of Average Refractive Index of Spin Coated DCG Films for HOE Fabrication
}

\author{
T. J. Kim, Eugene W. Campbell, and Raymond K. Kostuk \\ Department of Electrical and Computer Engineering \\ and Optical Sciences Center \\ University of Arizona \\ Tucson, AZ, 85721
}

\begin{abstract}
The refractive index of holographic emulsions is an important parameter needed for designing holographic optical elements (HOEs). In this paper theoretical calculations of the accuracy required for the refractive index and thickness of emulsions needed to meet predetermined Bragg angle conditions are presented. A modified interferometric method is used to find average refractive index of the unexposed and the developed dichromated gelatin holographic films. Slanted transmission HOEs are designed considering the index and thickness variations, and used to verify the index measurement results. The Brewster angle method is used to measure surface index of the unexposed and the developed films. The differences between average index and surface index are discussed. Theoretical calculation of the effects of index variation on diffraction efficiency, and experimental results for index modulation variation caused by process changes are also presented.
\end{abstract}

\subsection{Introduction}

The refractive index of dichromated gelatin (DCG) film is a function of many parameters. These include gelatin type, dichromate concentration, film thickness, environmental conditions (relative humidity and temperature), hologram construction conditions (exposure energy, construction geometry, wavelength) and chemical development. A number of researchers have previously investigated the refractive index of DCG films[1]-[8]. Several different techniques were used to investigate the index, such as Abbe refractometry, Brewster angle methods, interferometry with index matching oil, and diffraction efficiency measurements with coupled wave analysis. The reported index values range from 1.20 to 1.71 . This large uncertainty in index makes it difficult to accurately design holographic optical elements.

In order to design HOEs properly, the average refractive index before exposure and after development must be known within an acceptable range. This paper presents the experimental results for the surface and the average refractive index of spin coated DCG holographic films. It also shows how variations in these values affect the reconstruction angle for slanted volume holograms.

\subsection{The Effect of Index Change on Bragg Angle}

DCG film can swell or shrink after chemical development. This causes the grating $\underline{K}$ vector to rotate. The Bragg reconstruction angle (or angle for maximum diffraction efficiency) caused by a shift 
in the $\underline{\mathrm{K}}$ vector can be predicted using coupled wave analysis[9]. If the emulsion thickness during construction is $t_{c}$ and the thickness during reconstruction is $t_{r}$ a thickness factor can be defined as

$$
\mathrm{m}=\mathrm{t}_{\mathrm{r}} / \mathrm{t}_{\mathrm{c}}
$$

The $\underline{\mathrm{K}}$ vector will change according to

$$
\begin{array}{r}
\underline{K}_{r_{z}}=\frac{1}{m} \cdot \underline{K}_{c_{z}}, \\
\underline{K}_{r_{x}}=\underline{K}_{c_{x}} .
\end{array}
$$

where the subscripts $r$ and $c$ denote the reconstruction and construction, respectively. The slant angle $\phi$ will be modified as

$$
\tan \phi_{\mathrm{r}}=\mathrm{m} \cdot \tan \phi_{\mathrm{c}}
$$

From Bragg angle condition, $\cos (\phi-\varphi)=|\underline{K}| / 2 \beta[9]$, the reconstruction angle in the medium can be determined from

$$
\varphi_{\mathrm{r}}=\phi_{\mathrm{r}}+\cos ^{-1}\left(\frac{|\underline{\mathrm{K}}|}{2 \beta_{\mathrm{r}}}\right)-\pi
$$

where $\beta_{r}=2 \pi n_{r} / \lambda_{r}$. The shift in the Bragg reconstruction angle is a function of the thickness change $(\mathrm{m})$, and the average refractive index $\left(\mathrm{n}_{r}\right)$ after development. It varies with the value for the $\underline{K}$ vector slant angle as shown in Fig. $2\left(10^{\circ}\right)$, Fig. $3\left(30^{\circ}\right)$, and Fig. $4\left(50^{\circ}\right)$. Indices of the surface and the bulk area for the unexposed film were almost the same which were measured using the Brewster angle method (see 3.1) for the surface and using a modified interferometric method (see 3.2) for the bulk area.

When the $\underline{K}$ vector slant angle increases, the shift in Bragg reconstruction angle becomes larger in air for the same average refractive index variation. For example, when the desired reconstruction angle is $9.3^{\circ} \pm 0.5^{\circ}$, the predicted average refractive index of $n_{r}=1.44, n_{c}=1.59$, and the thickness swells by $5 \%(\mathrm{~m}=1.05)$, the allowed average refractive index can range from 1.27 to 1.60 (Fig.2). However, when the desired reconstruction angle is $45.4^{\circ} \pm 0.5^{\circ}$ with $\mathrm{n}_{r}=1.44, \mathrm{n}_{c}=1.59$, and $\mathrm{m}=1.05$, the allowed average refractive index range is reduced to 1.415 to 1.465 (Fig.4). Therefore, the range in average refractive index must be reduced for gratings with higher $\underline{\mathrm{K}}$ vector slant angles.

\subsection{Refractive Index Measurement Techniques}

All the DCG spin coated films used for these experiments have a composition consisting of [Ammonium dichromate : Gelatin : DI water $=10 \mathrm{gram}: 30 \mathrm{gram}: 250 \mathrm{ml}$. The film thickness was measured with a Tancor alpha-step 200 with a vertical resolution $0.005 \mu \mathrm{m}$. The unexposed DCG film is isotropic and uniform $\left(\mathrm{t}_{c} \pm 0.12 \mu \mathrm{m}\right)$ over $10 \mathrm{~mm}$. Thus, it is relatively easy to measure the refractive index. However, it is very difficult to measure the refractive index for the developed hologram because the film 
surface is not as uniform $\left(t_{r} \pm 0.32 \mu \mathrm{m}\right)$ and the phase variation through the grating is not linear[7], [8]. Therefore, several refractive index measuring techniques were used to evaluate developed DCG holographic gratings. The Brewster angle measurement technique and Fabry Perot resonant method are used to evaluate for the surface index, a modified interferometric method is used to determine the bulk average index of nongrating region, and the average index of grating region is measured using an analysis of the resultant grating efficiency.

\subsection{Brewster Angle Method}

This method (Fig.5) was developed by Pawluczyk et al. [4],[7],[8]. It gives only the surface refractive index[10]. Special care is required in measuring the Brewster angle because the interference of light reflected from air-emulsion and emulsion-substrate exists and it becomes dominant around the Brewster angle[4], [8].

The measurement results are, $\mathrm{n}_{c}(\lambda=514.5 \mathrm{~nm})=1.590 \pm 0.005, \mathrm{n}_{c}(\lambda=632.8 \mathrm{~nm})=1.570 \pm 0.005$ for the unexposed film and $n_{r}(\lambda=514.5 \mathrm{~nm})=1.570 \pm 0.005, \mathrm{n}_{r}(\lambda=6.32 .8 \mathrm{~nm})=1.540 \pm 0.005$ for the uniformly exposed and developed film.

\subsection{Interference Method}

This method is very useful for measuring the bulk index of the emulsion, however, the cutting ruins the film (Fig.6). Calibrated Cargille index matching oil is used and the remaining index matching oil between trials is removed with ethyl ether. There is some degree of error if the oil is not completely removed between measurements.

The film is rotated during the measurement by an angle $\theta_{e}$ (Fig. 6) in order to eliminate ambiguities in fringe matching. If the rotating angle inside the medium is $\theta_{e}$ and the index difference between emulsion and index matching oil is $\Delta \mathrm{n}$, then the relative fringe movement as a function of $\theta_{e}$ follows the relationship

$$
2 \cdot \frac{2 \pi \Delta \mathrm{nt}}{\lambda \cos \theta_{e}}=2 \pi(\mathrm{q}+\mathrm{I})
$$

where $\mathrm{t}$ is the film thickness, $\mathrm{q}$ is integer, and $\mathrm{I}$ is the fraction of relative fringe shift. When the shift completes one cycle by increasing the angle $\theta_{e}, I=1$, and it is possible to keep track of $|\Delta n|$ variation. When the index of applied matching oil is higher than that of the emulsion, the fringes will either shift up or down with increasing $\theta_{e}$. When the applied matching oil index is lower than that of the emulsion, the fringe shifts in the opposite direction as $\theta_{e}$ increases. However, Eq. (5) can not be applied in the holographic grating region since the phase variation through grating does not vary linearly with changing $\theta_{e}$. This method needs more improvements for the index measurement of gratings.

Measured values for the unexposed film were $\mathrm{n}_{c}(\lambda=6.32 .8 n \mathrm{~m})=1.58 \pm 0.01$, for the unexposed and developed film areas $n_{r}(\lambda=632.8 n m)=1.46 \pm 0.01$, and $n_{r}(\lambda=632.8 n m)=1.48 \pm 0.02$ for the uniformly exposed and developed film areas. 


\subsection{Grating Efficiency Analysis}

In this approach the shift in Bragg angle of a volume grating is compared to data using coupled wave analysis[9]. In previous work[6] polarization selective gratings were used to determine the index $\mathrm{n}_{r}$, and was found to be in a range from 1.2 to 1.4 . The specific value depends on the emulsion thickness and processing technique. This experiment was repeated in our lab with an interbeam construction angle of $2 \theta_{0}=30^{\circ}$ within the emulsion. This results gave an index $n_{r} \cong 1.38$.

By measuring the Bragg angle shift and thickness change factor $(\mathrm{m})$, the average refractive index can be deduced from the angular tolerance calculation as discussed in section 2.0. Transmission gratings with large slant angles (50 5 in air) were used since they produce larger shifts in Bragg angle. Pre and post processed film thickness measurements were obtained from different $2 \times 2.5$ inch $^{2}$ samples taken from the same $4 \times 5$ inch $^{2}$ plate. The plates were dehydrated with isopropanol/water concentrations of (25-50-75-100-100\%). The maximum efficiency of the hologram used for Bragg angle shift measurements was $88.4 \%$. The Bragg angle shift was determined from the change in the center of the FWHM values of the efficiency vs. angle measurements and found to change by $5^{\circ}$ (see the Exp. data curve in Fig. 7). This corresponds to an average index $n_{r}=1.360$ (Fig. 4).

Theoretical calculation based on the rigorous coupled wave analysis[11] was performed and compared with the experimental data in Fig.7. The measured thickness $\left(t_{r}\right)$ is $8.698 \mu \mathrm{m}$, however, the effective thickness for the curve fit is $5.0 \mu \mathrm{m}$. This lower effective thickness may be caused by the gradual change of index modulation with depth which may be caused by nonuniform chemical dehydration. The difference of index between surface (1.54) and the average value (1.46) is a good evidence for this. More research needs to be done to explain this effect.

\subsection{Fabry Perot Resonant Method}

Another method examined to determine the refractive index of the emulsion was by comparing the measured reflected beam intensity to calculated reflection values. In the case of a uniform thin emulsion on glass the interference of beam reflected from the air-emulsion and emulsion-glass surfaces give rise to a Fabry Pero effect[12]. Information from the modulated beam intensity can be used to determine the effective index of the emulsion. However, the DCG samples used in our experiment had a high level of surface reticulation in the exposed areas. This resulted in high levels of scatter from the air-emulsion surface which obscured the Fabry Perot effect.

In spite of these difficulties the average index of the emulsion surface could be determined, and was found to be 1.54 for a uniformly exposed region of film and 1.50 for a grating region at $632.8 \mathrm{~nm}$.

\subsection{Effect of Dehydration Rate on Index Modulation}

The index modulation depends on the exposed energy and the chemical process. Gradual dehydration with isopropyl alcohol concentration of (25-50-75-100-100\%) were compared to a rapid dehydration (50-100-100\%). 
The 1st peak of diffraction efficiency (DE) occurs with an exposure energy of about $70 \mathrm{~mJ} / \mathrm{cm}^{2}$ for the rapid dehydration and with approximately $220 \mathrm{~mJ} / \mathrm{cm}^{2}$ for the gradual dehydration. Normalized curves for DE vs. angle are given in Fig. 8. All measurements were performed as explained in 3.3. For rapid dehydration the Bragg angle shift is about $7.08^{\circ}$ and the thickness factor is about $m=1.07$. The resultant average refractive index $n_{r}$ is about 1.348. For the gradual dehydration process the Bragg angle shift is about $5.10^{\circ}$, and the thickness factor is about $\mathrm{m}=0.992$. This gives an average refractive index $n_{r}$ of about 1.355. The angular band width at full width - half maximum is $\Delta \theta \cong 13^{\circ}$ for the gradual dehydration process and $\Delta \theta \cong 9^{\circ}$ for the rapid dehydration. This implies that the effective thickness of the grating is greater for the rapidly dehydrated emulsion.

\section{$\underline{5.0 \text { Conclusions }}$}

In this report several techniques for measuring the change in the average refractive index were evaluated. The effect of inaccuracies in values used for the pre and post processed average index on the diffraction angle from transmission gratings were also determined.

The grating efficiency analysis method was the only method found which give consistent results for the effective index of holographic gratings. The surface refractive index for the holographic gratings was found to be considerably higher than the effective index (i.e. 1.50 vs 1.35). The effect of changing the rate dehydration did not appear to significantly change the effective refractive index.

\subsection{Acknowledgments}

The authors wish to thank the Optical Circuitry Cooperative at the University of Arizona and the National Science Foundation Grant No. ECS-9109954 for supporting this work. One of us (EWC) also wish to thank Eastman Kodak for a fellowship during this period.

\section{References}

[1] Shankoff, T. A., "Phase holograms in dichromated gelatin," Applied Optics, V7 N10, p2101, (1968).

[2] Meyerhofer, D., "Dichromated Gelatin," from a book Holographic recording materials, ed. by Smith, H. M., Springer-Verlag, Berlin Heidelberg, New York, p74 (1977).

[3] Newell, J. C. W., "Optical holography in dichromated gelatin," D. Phil. Thesis, Oxford University (1987).

[4] Pawluczyk, R., "Modified Brewster angle technique for the measurement of the refractive index of a DCG layer," Applied Optics, V29 N4, p.589 (1990).

[5] O'Connor, B., "Polarization properties of high numerical apeture holographic optical elements," MS Thesis, Univ. of Arizona (1989).

[6] Rallison, R. D. and Schicker, S. R., "Polarization properties of gelatin holograms," SPIE Vol. 1667, (1992). 
[7] Pawluczyk, R., et al., "Characterization of DCG holograms during the production process: some practical aspects," SPIE Vol. 954, Optical Testing and Metrology II, 40 (1988).

[8] Kim, T. J., "Optimization of dichromated film coatings for holographic recordings," MS Thesis, Univ. of Arizona (1991).

[9] Kogelnik, H., "Coupled wave theory for thick hologram gratings," Bell Sys. Tech. J., V48 N9, p2909 (1969).

[10] Hecht, E., "Optics," 2nd ed., Addison-Wesley Publishing Co., (1987).

[11] Gaylord, T. K., and Moharam, M. G., "Analysis and applications of optical diffraction by gratings," Proceedings of IEEE, V73 N5, p894 (1985).

[12] Yariv, A., "Optical Electronics," 4th ed., ch. 4, Holt, Rinehart and Winston Inc., Philadelphia, (1991). 


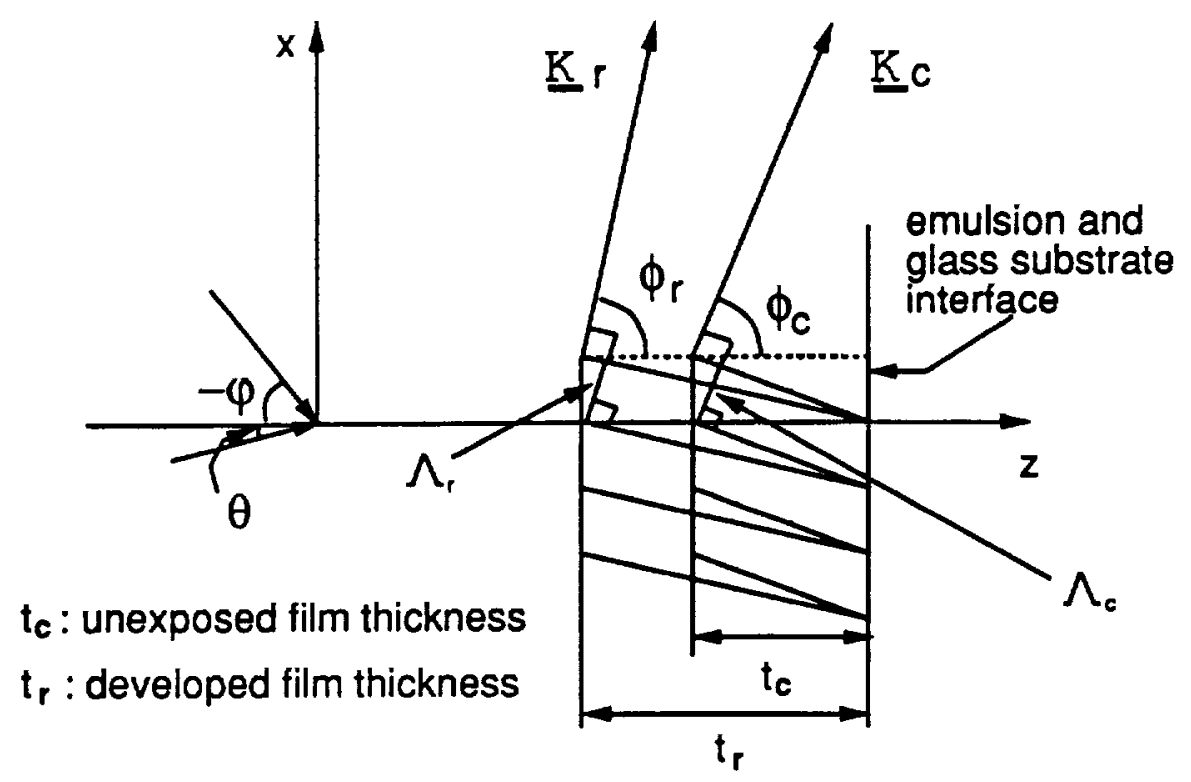

Figure 1: Schematic diagram for the $\underline{K}$ vector shift where $\mathbf{r}$ denotes reconstruction and $\mathrm{c}$ construction. $\Lambda_{r}$ and $\Lambda_{c}$ are grating spacings.

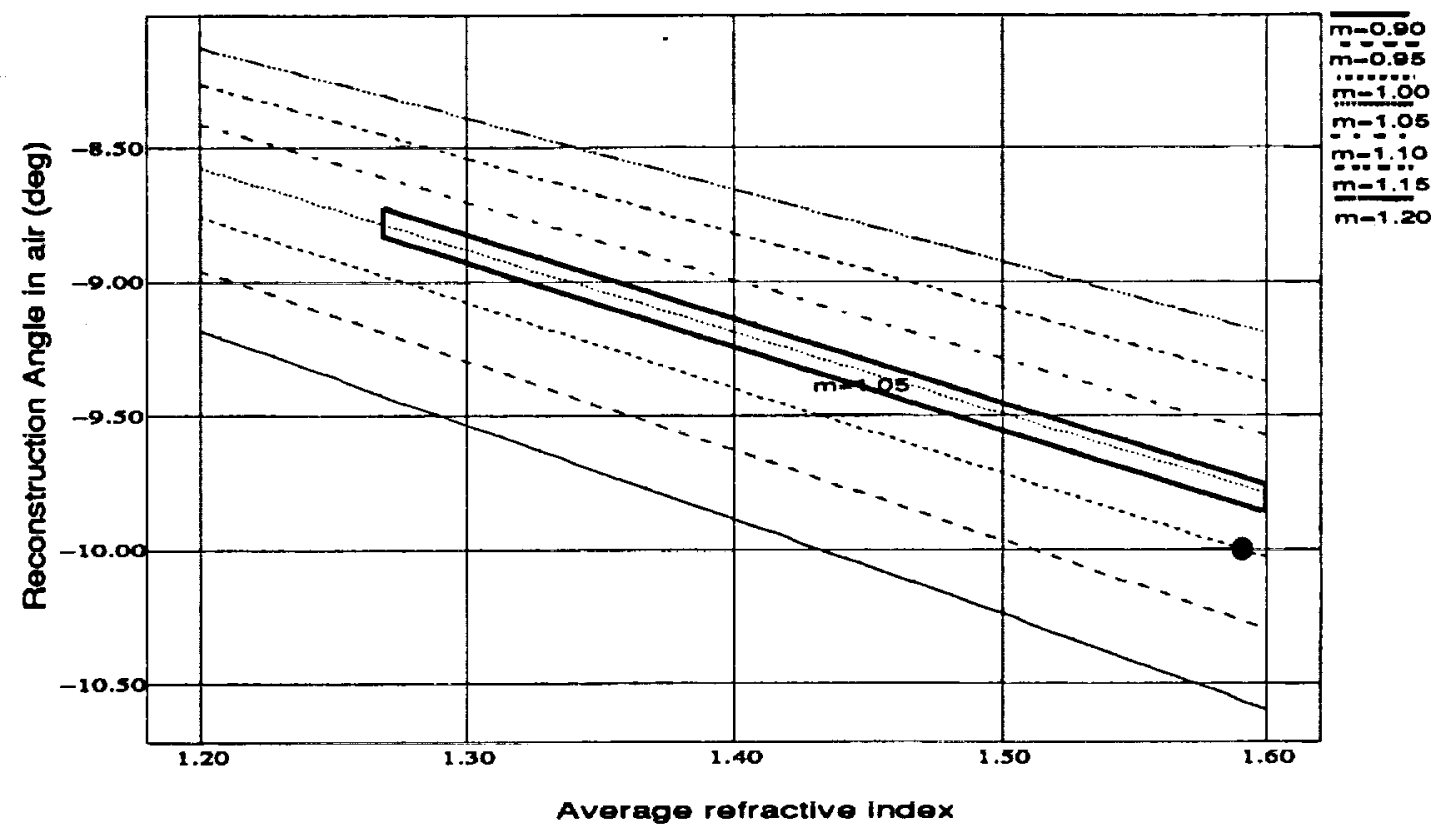

Figure 2: The Bragg angle shift with different thickness change factors as a function of average refractive index. Values given are for a slanted transmission grating; $\theta=0^{\circ},-\varphi=10^{\circ} \mathrm{in}$ air, $\lambda_{c}=\lambda_{r}=514.5 \mathrm{~nm}$. The point $\bullet$ denotes $\mathrm{n}_{c}=\mathrm{n}_{r}=1.59$ and no thickness variation. 


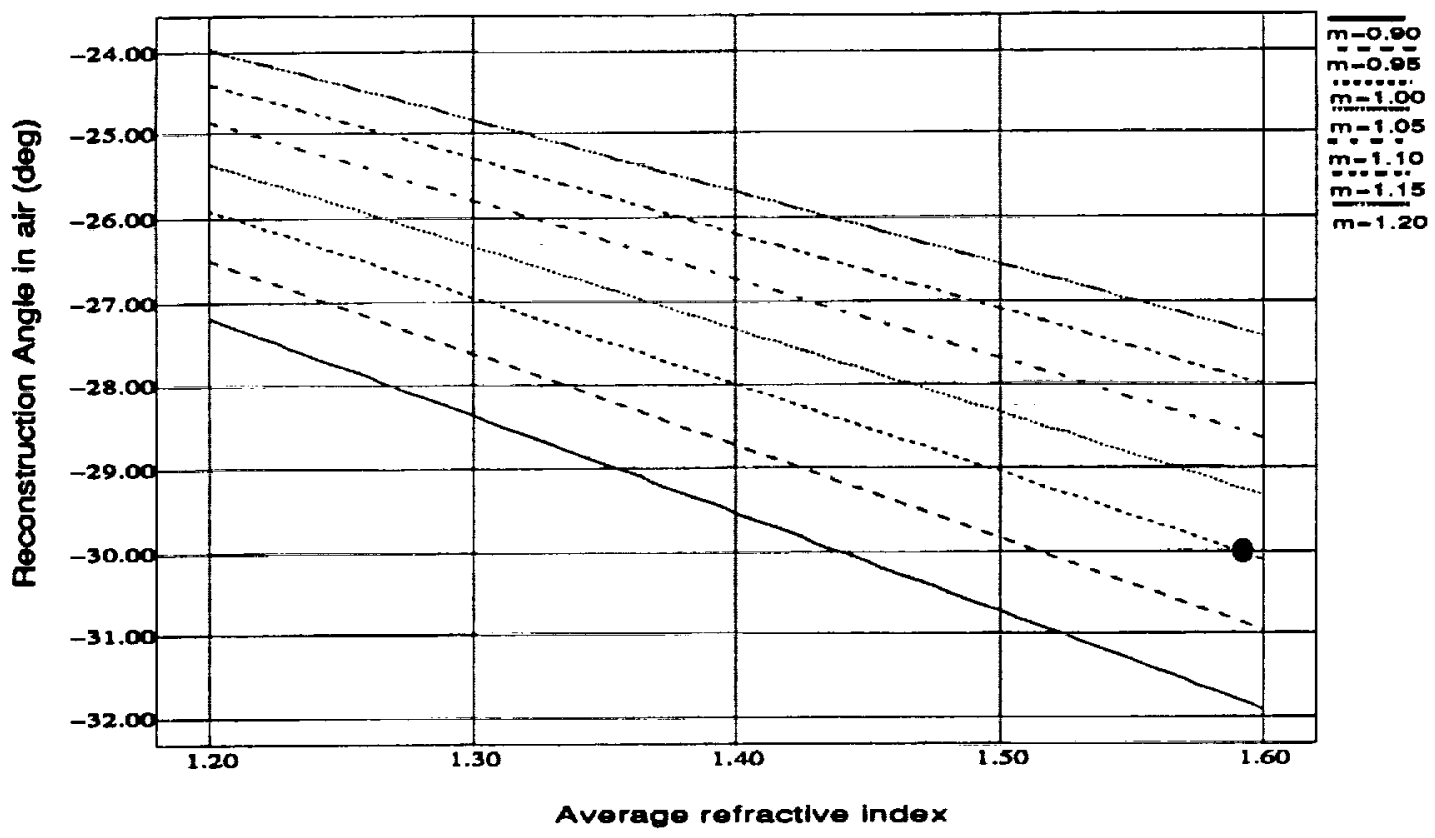

Figure 3: The Bragg angle shift with different thickness change factors as a function of average refractive index. Values given are for a slanted transmission grating; $\theta=0^{\circ},-\varphi=30^{\circ}$ in air, $\lambda_{c}=\lambda_{r}=514.5 \mathrm{~nm}$. The point $\bullet$ denotes $\mathrm{n}_{c}=\mathrm{n}_{r}=1.59$ and no thickness variation.

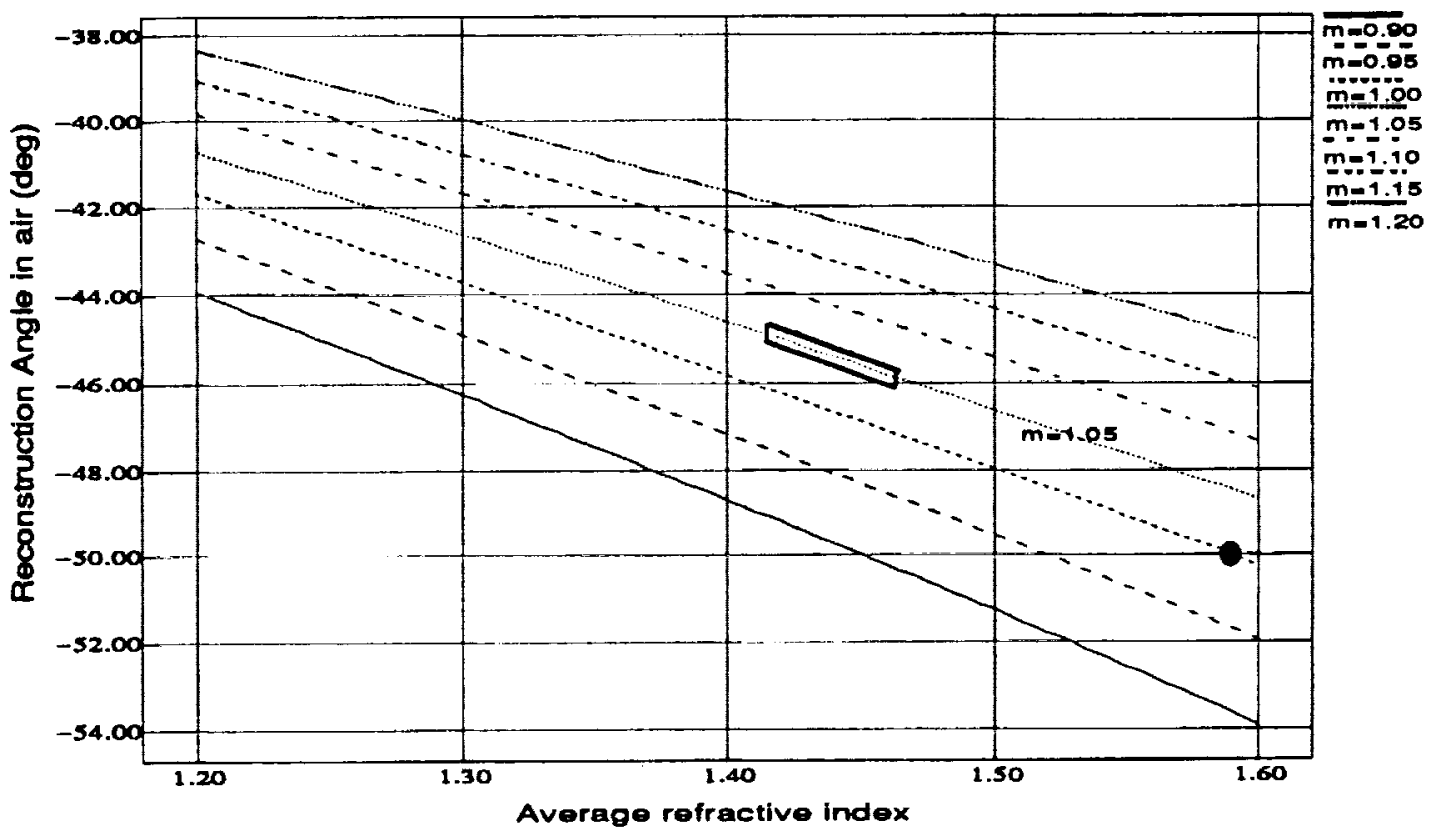

Figure 4: The Bragg angle shift with different thickness change factors as a function of average refractive index. Values given are for a slanted transmission grating; $\theta=0^{\circ},-\varphi=50^{\circ}$ in air, $\lambda_{c}=\lambda_{r}=514.5 \mathrm{~nm}$. The point $\bullet$ denotes $n_{c}=n_{r}=1.59$ and no thickness variation. 


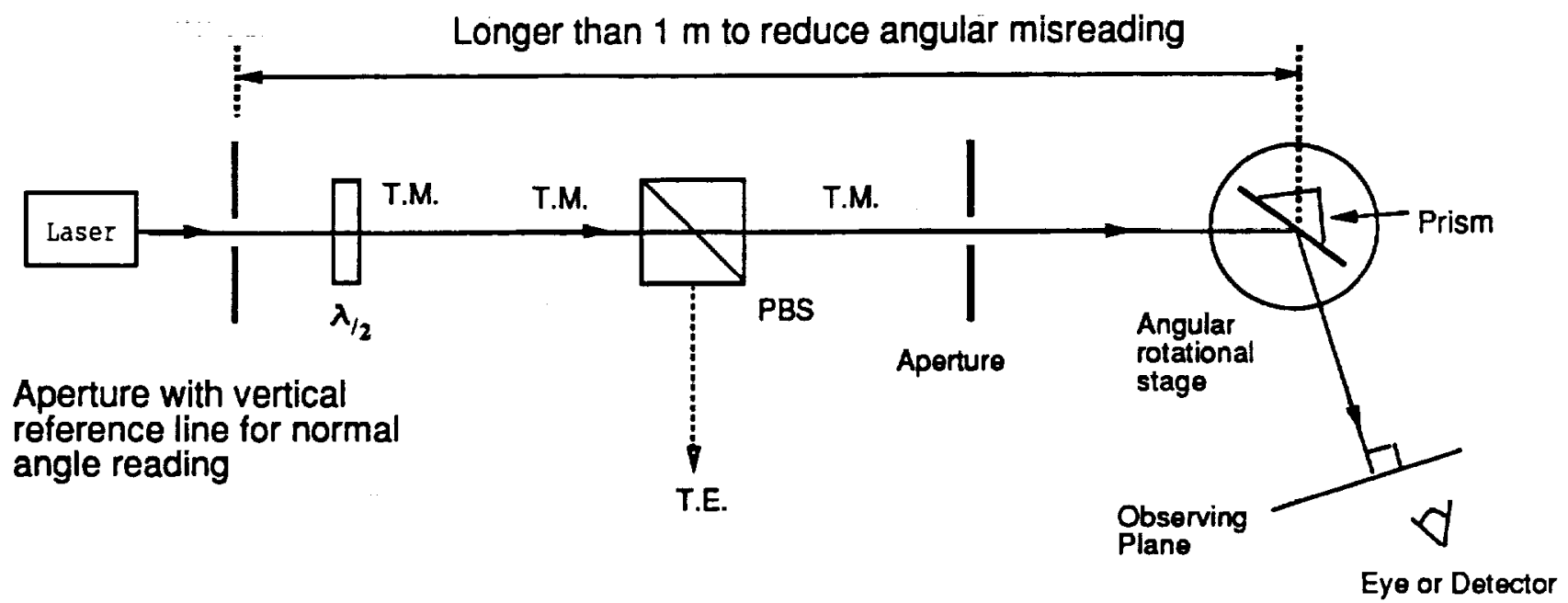

Figure 5: Optical set-up for Brewster angle measurement technique.

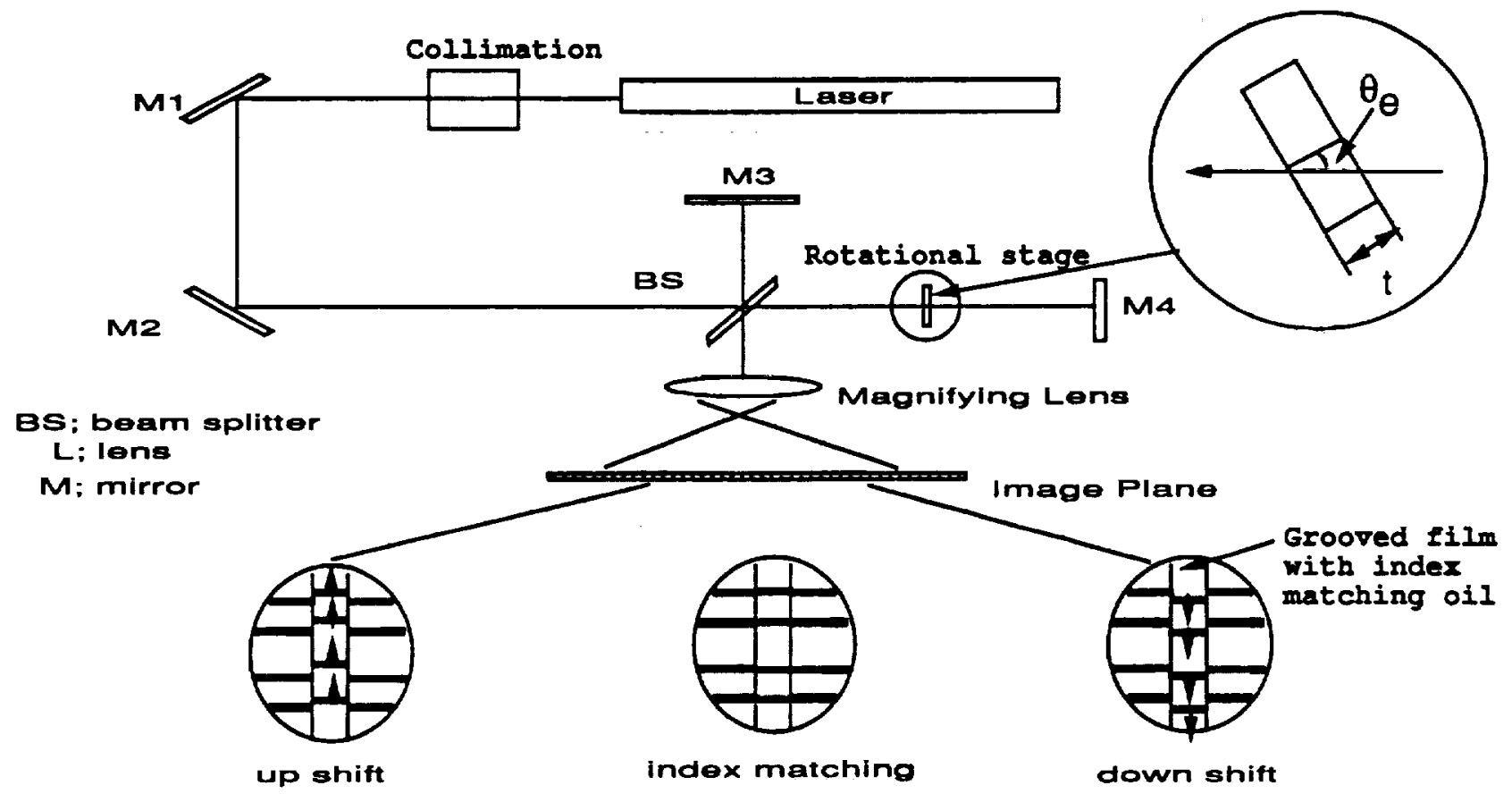

Figure 6: Optical set-up of modified interferometric technique for index matching measurement. 


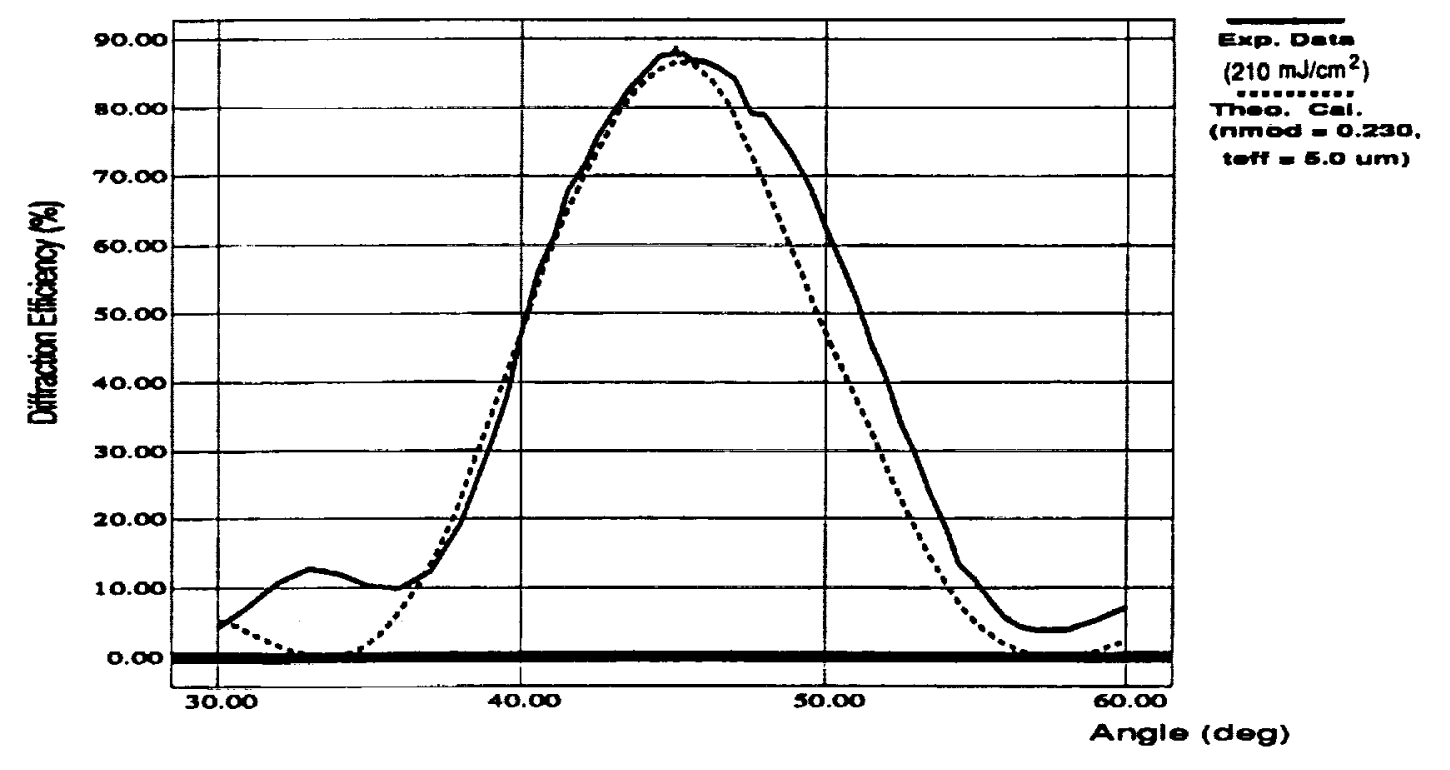

Figure 7: Comparison between experimental data and theoretical calculation. Values given are for a slanted transmission grating; $\theta=0^{\circ},-\varphi=50^{\circ}$ in air, $\mathrm{m}=0.998$; construction and reconstruction at $\lambda_{c}=\lambda_{r}=514.5 \mathrm{~nm}$.

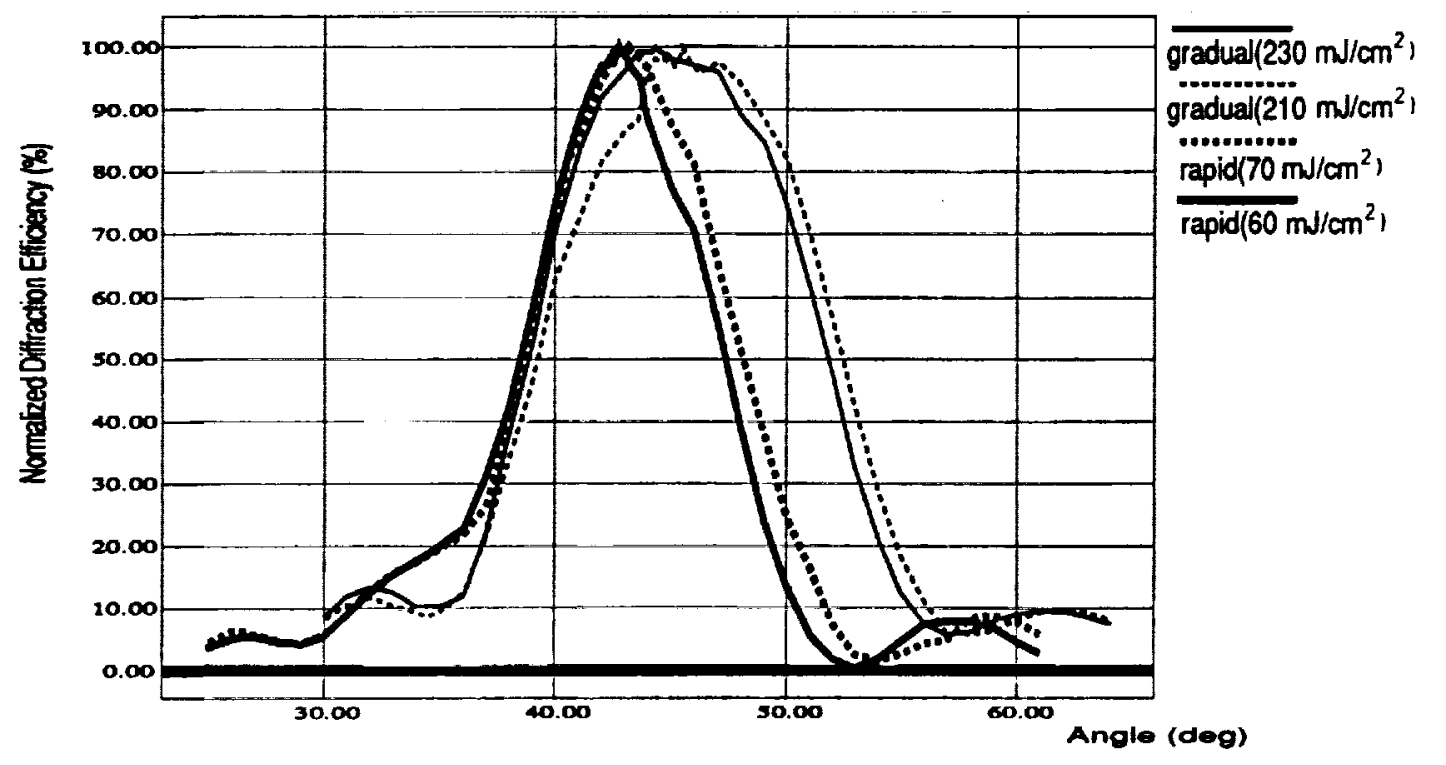

Figure 8: Experimental data of angular bandwidth for the comparison between rapid dehydration (Isopropyl alcohol concentration 50-100-100 \%) and gradual dehydration (25-50-75-100-100\%). The exposure energy to obtain maximum diffraction efficiency is about $70 \mathrm{~mJ} / \mathrm{cm}^{2}$ for the rapid dehydration process and about $230 \mathrm{~mJ} / \mathrm{cm}^{2}$ for the gradual dehydration process. Values given are for a slanted transmission grating; $\theta=0^{\circ},-\varphi=50^{\circ}$ in air; construction and reconstruction at $\lambda_{c}=\lambda_{r}=514.5 \mathrm{~nm}$. 
APPENDIX E

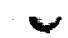

$\bullet$ 
$-$

$\nabla$

$\bar{\nabla}$

$\sigma$

$\sigma$

$\sigma$ 